Hernández Godoy, V.; Fernández Morales, K. y Pulido, J. (2018). La actitud hacia la educación en línea en estudiañtes universitarios. Revista de Investigación Educativa, 36(2), 349-364.

DOI: http://dx.doi.org/10.6018/rie.36.2.277451

\title{
La actitud hacia la educación en línea en estudiantes universitarios
}

\author{
Attitude towards e-learning in university students
Verónica Hernández Godoy*, Katiuska Fernández Morales ${ }^{* *}$ y Jesús Eduardo Pulido****
*Universidad Galileo. Instituto Von Neumann. Guatemala.
** Universidad Autónoma de Baja California. Instituto de Investigación y Desarrollo Educativo. México.
* Universidad Pedagógica Experimental Libertador. Venezuela.

\begin{abstract}
Resumen
Las instituciones educativas se enfrentan a una era de innovación constante. Diversas herramientas, metodologías de enseñanza-aprendizaje y sistemas virtuales ofrecen al estudiante la posibilidad de alcanzar sus metas, optimizar su desarrollo profesional y acceder a una educación de calidad. Tomar en cuenta la actitud de los estudiantes hacia la educación en línea es uno de los factores más importantes para diseñar, desarrollar e implementar iniciativas que permitan al estudiante aprovechar esta oferta educativa y obtener el mayor beneficio. Este artículo expone los resultados de la investigación que tiene como objetivo identificar los factores actitudinales que influyen en el aprendizaje en línea de los estudiantes universitarios. Este estudio es cuantitativo, no experimental descriptivo, ex-post facto. Se utilizó un cuestionario en línea que fue completado por 47 estudiantes de carreras universitarias de certificación, técnico, maestría y posgrado en educación en línea de la Universidad Galileo de Guatemala. Los resultados indican que los estudiantes con experiencia previa en tecnología y cursos en línea que reciben una educación con tutores preparados en un sistema fácil de utilizar y reciben adecuado soporte técnico y pedagógico sienten poco estrés ante los sistemas de aprendizaje en línea y tienen una actitud positiva hacia ellos.
\end{abstract}

Palabras clave: Educación en línea; actitud; estudiantes universitarios; aprendizaje.

Correspondencia: Verónica Hernández Godoy, verolucia74@gmail.com, Kilómetro 17.5 Carretera a El Salvador Lote 12 Arrazola 1, Guatemala. 


\begin{abstract}
Education institutions are facing an era of constant innovation. Various tools, teachinglearning methodologies, and virtual systems offer students the opportunity to achieve their educational goals, to optimize their professional development and to gain access to a quality education. To take into account the attitude of students towards online education is one of the most important factors to design, develop and implement initiatives enabling students to make use of this educational offer and get the most benefit. This article exposes the results of the research that aims to identify the attitudinal factors that influence the online learning of university students. The methodology in this study is quantitative, non-experimental descriptive ex-post facto. An online questionnaire was completed by 47 students aiming towards a certification, technical, master's degree and a graduate degree in online education of the Galileo University of Guatemala. The results indicated that the students with prior experience in technology and online learning which receive instruction from skilled tutors in a system easy to use and have the adequate technological and pedagogical support feel less stress about online learning and have a positive attitude towards it.
\end{abstract}

Keywords: Online Education; attitude; university students; learning.

\title{
Introducción
}

La educación en línea ha crecido rápidamente en los últimos años, una de las razones de este crecimiento es que representa una educación que no tiene fronteras, accesible a cada persona que desee aprender y para aquellos que por alguna razón no pueden acceder a la educación tradicional. Las universidades se esfuerzan por mantenerse al día con los avances tecnológicos, pero al mismo tiempo en ofrecer una alta calidad educativa con la cual tanto los estudiantes como las instituciones cumplan con los objetivos establecidos profesionalmente.

La introducción de la tecnología en la educación ha transformado el sistema. En consecuencia las personas también han modificado su comportamiento para adaptarse a nuevas metodologías de enseñanza-aprendizaje que demandan del estudiante motivación, responsabilidad, independencia y eficiencia en la comunicación. Por tanto, la decisión de iniciar o terminar un programa de educación en línea se ve afectada por diversos factores, uno de ellos es la actitud del estudiante.

Las actitudes se componen de componentes afectivos, cognitivos y conductuales, estos factores guían el gusto o disgusto de una persona hacia cierto objeto o conducta (Alabdullaziz, Muhammad, Alyahya \& Gall, s.f.). Aspectos como la seguridad en sí mismo, el uso de la tecnología, el género, estilo de aprendizaje y aspectos psicológicos y físicos influyen significativamente en la actitud del estudiante hacia el aprendizaje en línea, así como, la interfaz del programa, la facilidad de uso y la interacción (Chung, como se citó en Alabdullaziz et al., s.f.).

Según Bertea (2009) en el aprendizaje en línea una actitud positiva aumenta la probabilidad de que los estudiantes acepten los sistemas de educación. El éxito del aprendizaje depende de la actitud del aprendiz y ésta dará como resultado una participación activa o una resistencia pasiva (Lee \& Li, 2016). Para poder conocer cuál es la actitud de un estudiante hacia la educación en línea se utilizan escalas de acti- 
tud que de acuerdo a Elejabarrieta y Iñiguez (2000), son instrumentos que permiten conocer el componente afectivo de la persona hacia determinado objeto psicológico.

Ante los cambios anteriormente mencionados en el sistema educativo, las universidades en los países en vías de desarrollo como Guatemala están aumentando la oferta de cursos en línea para la población y así poder ampliar el acceso a la educación superior. A pesar del avance y el esfuerzo de las instituciones de educación por mantenerse al día, la Organización de las Naciones Unidas para la Educación, la Ciencia y la Cultura (UNESCO, 2013, p.6) en su estudio “Enfoques Estratégicos sobre las TICS en Educación en Latinoamérica y el Caribe" establece que, "La experiencia de incorporación de tecnologías en los sistemas educativos de América Latina y el Caribe en los últimos veinte años ha mostrado poco efecto en la calidad de la educación". Entre otras razones se debe a que se han introducido dispositivos y programas de computación sin estudios o estrategias que sustenten y apoyen esta nueva forma de enseñanza-aprendizaje. Lo anterior, ha llevado a que los resultados sean una práctica muy parecida a la que se utilizaba antes de la introducción de la tecnología de la información y comunicación (UNESCO, 2013).

Una de las razones de este rezago es que existe un vacío en los estudios en esta región acerca de la importancia de entender la actitud del estudiante hacia el aprendizaje en línea. El llevar a cabo investigaciones como esta permitirán que la educación en línea sea algo más que asistir la educación a través de la tecnología y se convierta en una modalidad de aprendizaje respaldada con fundamentos pedagógicos, metodológicos y una adecuada comprensión de los factores humanos que motivan la conducta del estudiante.

Los países en desarrollo tienen dificultad para mantenerse al día con los avances vertiginosos de la tecnología educativa y la implementación de mejores prácticas basadas en investigación (Yaghoubi, Mohammadi, Irivani, Attaran \& Gheidi, 2008), para progresar es de vital importancia tomar en cuenta qué factores motivan al estudiante a llevar cabo un cambio de la educación tradicional a una educación en línea de calidad.

Morales (2012), en el artículo: “E-Learning en Guatemala, Perspectivas y Oportunidades para Instituciones Educativas" afirma que, en Guatemala existen 13 universidades privadas y una universidad pública y todas poseen un LMS (Learning Managment System). El 23\% de universidades ofrece cursos libres $100 \%$ virtuales, orientados especialmente a los catedráticos. Algunas universidades nacionales ofrecen carreras de posgrado y maestrías en modalidad 100\% virtual para los estudiantes. La oferta de universidades internacionales se suma a la de instituciones nacionales.

A pesar de que, la oferta de educación en línea en Guatemala aumenta rápidamente, así como los usuarios de internet, poco o ningún esfuerzo conocido se ha realizado hasta el momento para entender la importancia de la actitud del estudiante hacia la educación en línea y cómo esto influye en la participación, satisfacción y permanencia del estudiante en este tipo de programas. Según Holley (como se citó en Mothibi, 2015) los estudiantes universitarios que participan de la educación en línea logran un mejor rendimiento académico comparado con universidades que utilizan métodos tradicionales.

Tomando en cuenta lo antes mencionado es claro que la educación en línea continuará expandiéndose y desarrollándose. Atendiendo a las variables que influyen en la actitud del estudiante hacia la educación en línea se puede lograr que cada vez más personas aprovechen esta oportunidad educativa. Además del contenido del curso es de 
vital importancia, el diseño de la plataforma de aprendizaje, considerando los aspectos tecnológicos e interactivos, como la percepción de la facilidad de uso, la flexibilidad, la asistencia, la actitud del tutor, etc. (Liaw, como se citó en Alabdullaziz, et al., (s.f.)).

Según el modelo de aceptación de la tecnología (TAM) el cual indica cómo los usuarios llegan a aceptar una tecnología; la utilidad percibida y la facilidad de uso son dos factores importantes que influyen en cómo los usuarios van a utilizar una tecnología y cuándo lo van a hacer. La utilidad percibida de un objeto se refiere a cómo cree el usuario que una tecnología nueva lo va a ayudar a aumentar su desempeño y la facilidad de uso se refiere a cuán libre de dificultad es el uso de la nueva tecnología (Orantes, 2011). Ambas impactan la actitud del usuario hacia la tecnología y es por esto que la mayor parte de cuestionarios que pretenden medir la actitud de los estudiantes hacia la educación en línea trabajan con estas dos variables. Según Orantes (2011), el usuario elegirá entre dos sistemas con funciones iguales aquel con el que le sea más fácil trabajar.

Ante esta vertiginosa transformación educativa hay algo que los educadores conocen desde hace mucho tiempo y es que, existe una estrecha conexión entre la actitud y la conducta. Ajzen (1991), se ha dedicado ampliamente a estudiar cómo la actitud influye en las decisiones de las personas de participar en ciertos comportamientos. Desarrolló la teoría de la conducta planeada la cual explica que la actitud se refiere al sentimiento positivo o negativo de un estudiante para llevar a cabo determinada conducta. La conducta son las acciones que el individuo lleva a cabo y pueden ser planeadas o deliberadas. A través de extensivos estudios concluyó que cuando las personas planifican su conducta se puede predecir con bastante precisión su intención, pero solo si se tiene comprensión de tres factores principales: la actitud (evaluación de ideas, eventos y objetos) el control que perciba que tiene sobre su conducta (la dirección de sus acciones) y las normas (actitudes y conductas típicas). Mientras más positiva sea la actitud más fuerte será la intención pues, la conducta y esfuerzo son dirigidos por la motivación. En otras palabras, para saber qué hará la persona es importante saber qué desea hacer, es por esto que entender lo que espera el estudiante obtener de la educación en línea es indispensable para poderle proveer de una instrucción que cubra sus necesidades y aumente su satisfacción.

Para lograr una relación positiva entre el estudiante y el aprendizaje en línea es necesario conocer cómo deber ser diseñada, impartida y distribuida la instrucción de acuerdo a las necesidades de los estudiantes y las variables que influyen en su actitud.

El aprendizaje en línea es una opción que representa ventajas para el estudiante como, flexibilidad y conveniencia, accesibilidad a estudiantes diversos, pero también posee desventajas que suelen estar relacionadas con el uso o disponibilidad de la tecnología. Un estudiante que participa de esta modalidad de aprendizaje debe poseer competencias digitales básicas como comunicación por e-mail, habilidad para manejar buscadores y software de escritura (Bertea, 2009). Cuando el estudiante percibe que no tiene la experiencia o habilidad tecnológica básica puede sentirse ansioso hacia la educación en línea y la falta de seguridad y familiaridad con los sistemas afecta su actitud y por consiguiente su desempeño académico. Otros estudios indican que la falta de interacción humana y para algunos la falta de un tutor que monitoree de forma presencial el desempeño del estudiante puede afectar también la actitud del estudiante. 
La educación en línea es una modalidad de enseñanza-aprendizaje que integra la tecnología de la información y la comunicación. Su desarrollo ha provocado cambios importantes en el sistema educativo. Bertea (como se citó en Nichols, 2003), define la educación en línea como el uso de varias herramientas tecnológicas que son basadas en la red, distribuidas por la red o apropiadas para la red con propósitos educativos. El aprendizaje en línea ofrece una educación flexible para aquellos que no pueden acceder a la educación por limitaciones de tiempo, ubicación, discapacidad o simplemente por conveniencia, además de ser una opción para aquellos que disfrutan de aprender a través de la tecnología educativa. Con la introducción y crecimiento de la educación virtual las instituciones de educación superior tienen la oportunidad de ofrecer la flexibilidad, eficiencia y accesibilidad geográfica y económica que la educación en línea promete; un sistema centrado en el estudiante que lo estimula a construir el propio conocimiento a través de la reflexión, en el tiempo y espacio que le sea más conveniente.

Es por esto que las instituciones de educación superior trabajan por adaptar las TIC en sus sistemas educativos, según Majo y Márquez (como se citó en Centeno \& Cubo, 2013) existen tres reacciones posibles para dicha adaptación: en el primer escenario, el tecnócrata; las instituciones realizan cambios pequeños como introducir la alfabetización digital, primero se aprende SOBRE las TIC y DE las TIC. El siguiente escenario es el reformista en el cual se cumplen los dos niveles anteriores, aprender SOBRE las TIC y DE las TIC y se introduce el aprender CON las TIC. En el último escenario que es el holístico se reestructuran todos los elementos con base en las TIC.

Aún y con estas adaptaciones hacia la introducción de la tecnología educativa, las instituciones de educación superior se ven obligadas a ir más allá de su inclusión para el aprendizaje. Un estudiante que no se siente cómodo con el sistema con el cual pretende aprender tiene escasas posibilidades de continuar sus estudios. Brindar atención al componente afectivo que motiva a los estudiantes a estudiar en línea permite planificar estratégicamente cursos e iniciativas que lleven al estudiante a permanecer motivado y obtener un desempeño académico satisfactorio que lo conduzca a cumplir sus objetivos de aprendizaje.

Es por lo anterior que es importante realizar una primera aproximación en Guatemala preguntando en este trabajo de investigación: ¿Cuáles son las actitudes predominantes en los estudiantes universitarios en la educación en línea?

Esta investigación se realizó con el objetivo de identificar los factores actitudinales que influyen en los estudiantes de educación en línea y con los resultados obtenidos poder aportar datos que contribuyan a mejorar el diseño, planificación e iniciativas institucionales de aprendizaje virtual.

\section{Revisión de investigaciones similares}

La medición de actitudes hacia la educación en línea ha sido estudiada desde diversas metodologías y contextos en una variedad de estudios. Un estudio realizado por Pilli, Fanaeian y Al-Momani (2014) midió las actitudes de los estudiantes hacia el aprendizaje en línea a través de una escala de actitudes la cual fue completada por estudiantes que ya tenían familiaridad con la educación en línea. Los autores concluyeron que los resultados son consistentes con estudios anteriores que indican que la calidad 
del sistema influye en la satisfacción personal del estudiante y la utilidad percibida de los sistemas de aprendizaje en línea. La utilidad percibida por el estudiante tiene una fuerte influencia en su actitud hacia la educación en línea y la facilidad de uso tiene una influencia más moderada sobre la actitud del estudiante, aunque ambos factores afectan las actitudes hacia el aprendizaje en línea. Finalmente encontraron que los estudiantes de la Universidad de Girne están interesados en el uso del sistema de educación en línea que les provea de información de calidad y mayor productividad en sus tareas.

En otro estudio realizado por Paris (2004), en el cual se estudiaron las actitudes de estudiantes de secundaria hacia el aprendizaje asistido por internet se encontró que la mayoría de estudiantes preferían la educación asistida por internet para publicar y utilizar diagramas, gráficas, animaciones y vídeos. Según sus conclusiones los estudiantes se sentían atraídos y más motivados al utilizar elementos multimedia en la educación. También encontró diferencias entre las tareas preferidas por hombres y mujeres indicando que los hombres se sentían mayormente atraídos por tareas que proveen un estímulo inmediato (animación, sonido, juegos, etc.) y las mujeres se sentían más atraídas hacia descargar elementos que podían serles útiles en el futuro.

Saabah (2013), investigó los factores que afectan la aceptación de la educación en línea a través de un estudio de la motivación y actitudes de los estudiantes hacia el aprendizaje en línea. Los sujetos del estudio fueron 100 estudiantes de pregrado, elegidos al azar de una universidad en Palestina. Las conclusiones del estudio fueron que los estudiantes le prestaron poca importancia a beneficios como el manejo del tiempo, flexibilidad y reducción de costos y se encontró una correlación positiva entre las habilidades técnicas del estudiante y su actitud hacia el aprendizaje en línea. Los estudiantes con poca experiencia con la tecnología o sin experiencia previa con la educación en línea estaban poco motivados hacia el aprendizaje en línea y no tenían consciencia de sus beneficios.

Existen también estudios que han relacionado la actitud con el desempeño académico en estudiantes de educación en línea. Oye, A.Iahad, Madar y Ab. Rahim, (2012), estudiaron el impacto de la educación en línea en el desempeño académico concluyendo que, existe una correlación positiva entre el desempeño académico de los estudiantes y aprendizaje en línea, pero, para que el estudiante tenga la intención de utilizarlo debe tener una percepción positiva acerca de su uso. Además, refieren que, "mientras las actitudes tienen una influencia sobre la intención de uso, el uso en sí de la educación en línea tiene un efecto significativo en el desempeño de los estudiantes" (p.129).

Mothibi (2015) en un estudio similar refirió que el uso adecuado de las tecnologías de la información y la comunicación en la educación en línea son una herramienta efectiva para mejorar el rendimiento de los estudiantes.

Los anteriores estudios son consistentes en indicar que si los estudiantes se sienten cómodos con la tecnología y sienten que obtienen un beneficio directo de un sistema de educación que perciben como eficiente e interactivo, van a tener una mejor actitud hacia el aprendizaje en línea pues se encuentran motivados.

Bertea (2009) en un estudio de casos midió la actitud de los estudiantes universitarios hacia la educación en línea. Se midieron las actitudes, las habilidades tecnológicas y se definió el perfil demográfico y social. Los resultados obtenidos revelaron una actitud positiva de los estudiantes hacia la educación en línea con mayor positivismo expresado a través de los estudiantes que trabajan en comparación con aquellos que no trabajan. 
Los estudiantes con empleo, sin embargo, expresaron que es necesario una preparación tecnológica más especializada a pesar de tener experiencia con las computadoras y facilidad en utilizar el software educativo. Se encontró una correlación pobre entre la actitud del estudiante y las habilidades técnicas, los estudiantes que utilizan con mayor frecuencia una computadora es más probable que acepten la educación en línea. Una de las limitantes del estudio fue que los estudiantes no utilizaban un sistema de educación virtual y la información se recopiló de acuerdo a su conocimiento acerca de la educación en línea.

También se han realizado esfuerzos por desarrollar instrumentos que midan la actitud del estudiante hacia la educación en línea. Mehra y Omidian (2012) desarrollaron un instrumento con este fin con mediciones en percepción de utilidad, intención para utilizar e-learning, facilidad de uso, soporte pedagógico y técnico, estresores y presiones para usar e-learning.

En cuanto a Latinoamérica en México, Padilla y Rodríguez (2002), estudiaron la actitud hacia la educación en línea y la experiencia previa con un curso en línea. Concluyeron que los estudiantes mostraron una actitud positiva hacia la educación en línea y que no estaba influenciada por su experiencia previa en un curso en línea, pero sí por experiencia previa con el uso de la tecnología.

\section{Método}

\section{Tipo de investigación}

Esta investigación es de tipo cuantitativa, descriptiva no experimental ex post-facto. Es también un estudio de tipo transversal, debido a que los datos fueron recuperados en un momento único a través de una encuesta (Hernández, Fernández \& Baptista, 2014). Las variables investigadas incluyen el aprendizaje en línea y la actitud.

\section{Población y muestra}

Participaron 47 estudiantes de pregrado y posgrado de educación en línea de la Universidad Galileo de Guatemala. La universidad cuenta con carreras de certificación, técnico, posgrado y maestría en educación en línea impartidas en modalidad 100\% virtual. En esta investigación se recabó la perspectiva del estudiante como usuario final. Los estudiantes fueron seleccionados en un muestreo por conveniencia no probabilístico. En este tipo de estudios se selecciona la muestra de forma no aleatoria con características similares a la de la población objetivo. Los resultados son únicos a la muestra evaluada (Casal \& Matéu, 2003).

\section{Instrumento}

Para el estudio se utilizó un cuestionario que mide la actitud de los estudiantes hacia la modalidad de educación en línea, traducida y adaptada de su versión original al español. El instrumento original estaba compuesto de 83 preguntas y fue desarrollado para medir la actitud de los estudiantes universitarios hacia la educación en línea de India e Irán. 
En sus estudios preliminares se descartaron 9 ítems de 92 iniciales después de medir la consistencia interna con Alpha Cronbach. La confiabilidad del instrumento original fue medida como muy buena al obtener un puntaje total de .834 (Mehra \& Omidian, 2012).

Para este estudio se elaboró un instrumento inicial que contenía las 83 preguntas del cuestionario original, luego de la revisión por un panel de expertos resultaron 76 preguntas. Se determinó la consistencia interna del instrumento para determinar hasta qué punto el ítem es relevante y representa el dominio actitudinal que se pretende medir. Para la revisión de los ítems se utilizó el Alfa de Cronbach y basado en los resultados se eliminaron 19 ítems que presentaron un índice de correlación negativo. La validez fue evaluada a juicio de expertos que realizaron observaciones y se realizaron correcciones. Después de eliminar los 19 ítems con índice de correlación negativa el instrumento quedó compuesto de 57 preguntas.

La escala para medir la actitud hacia la educación en línea obtuvo un resultado de confiabilidad de consistencia interna alta (0.795), según Ruiz-Bolívar (2002), quien sostiene que cuando el índice de confiabilidad está en el rango 0.61 a 0.80 la confiabilidad es alta (p. 70).

Para el estudio de la actitud se utilizó una escala de Likert con cinco respuestas posibles que van desde muy de acuerdo hasta muy en desacuerdo con un punto medio neutro.

\section{Procedimiento de recogida y análisis de datos}

Los estudiantes fueron contactados por la universidad a través de correo electrónico. Cada estudiante completó el cuestionario en línea. El estudio fue realizado durante el mes de noviembre de 2016 y se cerró al recibir 47 respuestas.

La información recolectada en la escala se analizó mediante el procedimiento de tablas cruzadas (realizado con SPSS), el cual permitió asociar el género, el número de cursos tomados en línea y los cinco factores analizados en este estudio para lograr evidenciar el acuerdo, desacuerdo e indiferencia en cada uno de los cinco factores los cuales estaban distribuidos en el cuestionario de la siguiente manera: 28 preguntas de percepción de utilidad, 9 preguntas de intención de utilizar el aprendizaje en línea, 5 preguntas de facilidad de uso, 8 preguntas de soporte pedagógico y técnico y 7 preguntas de estresores del aprendizaje virtual. Además, el cuestionario contiene en su inicio cuatro preguntas de perfil demográfico. En el cuestionario en línea se utilizó el término e-learning para referirse a la educación en línea por ser el término con el que están familiarizados los estudiantes y ser el utilizado por la universidad para identificar las carreras de educación en línea.

\section{Resultados}

De la población de 47 estudiantes que completaron el cuestionario, $57.4 \%$ de los estudiantes son mujeres y el 42.5 hombres. El 63\% de los estudiantes tomó más de 3 cursos en línea y el $36.1 \%$ tomó entre uno y tres cursos. La mayor parte de los estudiantes reportaron tener 46 años y más, seguidos por el grupo de 26 a 35 años y el de 36 a 45 . Solamente el $12.5 \%$ de los estudiantes reportaron ser de 25 años o menores (ver tabla 1). 
Tabla 1

Distribución del nivel de acuerdo de los estudiantes hacia la percepción de utilidad de la educación en línea

\begin{tabular}{|c|c|c|c|c|c|}
\hline \multirow{2}{*}{ Género } & & & \multicolumn{2}{|c|}{$\begin{array}{c}\text { ¿Cuántos cursos ha tomado } \\
\text { en línea? }\end{array}$} & \multirow{2}{*}{ Total } \\
\hline & & & $\begin{array}{c}\text { De } 1 \text { a } 3 \\
\text { cursos }\end{array}$ & $\begin{array}{c}\text { Más de } 3 \\
\text { cursos }\end{array}$ & \\
\hline \multirow{8}{*}{ Masculino } & \multirow{6}{*}{$\begin{array}{l}\text { Percepción } \\
\text { de utilidad }\end{array}$} & De acuerdo & 3 & 6 & 9 \\
\hline & & De acuerdo & $50.0 \%$ & $42.9 \%$ & $45.0 \%$ \\
\hline & & Ni de acuerdo ni en & 2 & 8 & 10 \\
\hline & & desacuerdo & $33.3 \%$ & $57.1 \%$ & $50.0 \%$ \\
\hline & & & 1 & 0 & 1 \\
\hline & & Muy en desacuerdo & $16.7 \%$ & $0.0 \%$ & $5.0 \%$ \\
\hline & \multirow{2}{*}{ Total } & & 6 & 14 & 20 \\
\hline & & & $100.0 \%$ & $100.0 \%$ & $100.0 \%$ \\
\hline \multirow{8}{*}{ Femenino } & \multirow{6}{*}{$\begin{array}{l}\text { Percepción } \\
\text { de utilidad }\end{array}$} & De acuerdo & 9 & 8 & 17 \\
\hline & & De acuerdo & $81.8 \%$ & $50.0 \%$ & $63.0 \%$ \\
\hline & & Ni de acuerdo ni en & 2 & 7 & 9 \\
\hline & & desacuerdo & $18.2 \%$ & $43.8 \%$ & $33.3 \%$ \\
\hline & & Muv en desacuerdo & 0 & 1 & 1 \\
\hline & & & $0.0 \%$ & $6.3 \%$ & $3.7 \%$ \\
\hline & \multirow{2}{*}{ Total } & & 11 & 16 & 27 \\
\hline & & & $100.0 \%$ & $100.0 \%$ & $100.0 \%$ \\
\hline \multirow{8}{*}{ Total } & \multirow{6}{*}{$\begin{array}{l}\text { Percepción } \\
\text { de utilidad }\end{array}$} & De acuerdo & 12 & 14 & 26 \\
\hline & & De acuerdo & $70.6 \%$ & $46.7 \%$ & $55.3 \%$ \\
\hline & & Ni de acuerdo ni en & 4 & 15 & 19 \\
\hline & & desacuerdo & $23.5 \%$ & $50.0 \%$ & $40.4 \%$ \\
\hline & & Muv en desacuerdo & 1 & 1 & 2 \\
\hline & & & $5.9 \%$ & $3.3 \%$ & $4.3 \%$ \\
\hline & \multirow{2}{*}{\multicolumn{2}{|c|}{ Total }} & 17 & 30 & 47 \\
\hline & & & $100.0 \%$ & $100.0 \%$ & $100.0 \%$ \\
\hline
\end{tabular}

De la totalidad de alumnos que tomaron de 1 a 3 cursos en línea tanto los hombres $(50 \%)$ como las mujeres ( $82 \%$ aproximadamente), manifestaron acuerdo con la utilidad de la educación en línea pues consideran que ayuda a resolver algunos problemas educativos, brinda nuevas oportunidades para la enseñanza-aprendizaje, ahorra tiempo y esfuerzo, además, el acceso a la educación aumenta a través del aprendizaje en línea, así como la flexibilidad de la enseñanza-aprendizaje y también ayuda a mejorar la capacidad de razonamiento lo que permite al estudiante ser más eficiente y productivo en los cursos. Con respecto a los que tomaron más de 3 cursos en línea, se determinó que alrededor del $43 \%$ de los alumnos y $50 \%$ de las alumnas manifestaron acuerdo con la utilidad de la educación en línea en los aspectos antes mencionados; pese al grado de acuerdo de algunos estudiantes hay un porcentaje importante de hombres y mujeres $57 \%$ y $43.8 \%$ respectivamente que no están ni de acuerdo ni en desacuerdo con la utilidad de la educación en línea (ver tabla 2). 
Tabla 2

Distribución del nivel de acuerdo de los estudiantes hacia la intención de adoptar la educación en línea

\begin{tabular}{|c|c|c|c|c|c|}
\hline \multirow{2}{*}{ Género } & & & \multicolumn{2}{|c|}{$\begin{array}{c}\text { ¿Cuántos cursos ha tomado en } \\
\text { línea? }\end{array}$} & \multirow{2}{*}{ Total } \\
\hline & & & $\begin{array}{c}\text { De } 1 \text { a } 3 \text { cur- } \\
\text { sos } \\
\end{array}$ & $\begin{array}{l}\text { Más de } 3 \\
\text { cursos }\end{array}$ & \\
\hline \multirow[t]{3}{*}{ Masculino } & \multirow[t]{2}{*}{$\begin{array}{l}\text { Intención de adoptar } \\
\text { elearning }\end{array}$} & $\begin{array}{l}\text { Muy de } \\
\text { acuerdo }\end{array}$ & $\begin{array}{c}4 \\
66.7 \%\end{array}$ & $\begin{array}{c}14 \\
100.0 \%\end{array}$ & $\begin{array}{c}18 \\
90.0 \%\end{array}$ \\
\hline & & De acuerdo & $\begin{array}{c}2 \\
33.3 \%\end{array}$ & $\begin{array}{c}0 \\
0.0 \%\end{array}$ & $\begin{array}{c}2 \\
10.0 \%\end{array}$ \\
\hline & Total & & $\begin{array}{c}6 \\
100.0 \%\end{array}$ & $\begin{array}{c}14 \\
100.0 \%\end{array}$ & $\begin{array}{c}20 \\
100.0 \%\end{array}$ \\
\hline \multirow[t]{3}{*}{ Femenino } & \multirow[t]{2}{*}{$\begin{array}{l}\text { Intención de adoptar } \\
\text { elearning }\end{array}$} & $\begin{array}{l}\text { Muy de } \\
\text { acuerdo }\end{array}$ & $\begin{array}{c}10 \\
90.9 \%\end{array}$ & $\begin{array}{c}15 \\
93.8 \%\end{array}$ & $\begin{array}{c}25 \\
92.6 \%\end{array}$ \\
\hline & & De acuerdo & $\begin{array}{c}1 \\
9.1 \%\end{array}$ & $\begin{array}{c}1 \\
6.2 \%\end{array}$ & $\begin{array}{c}2 \\
7.4 \%\end{array}$ \\
\hline & Total & & $\begin{array}{c}11 \\
100.0 \%\end{array}$ & $\begin{array}{c}16 \\
100.0 \%\end{array}$ & $\begin{array}{c}27 \\
100.0 \%\end{array}$ \\
\hline \multirow[t]{3}{*}{ Total } & \multirow[t]{2}{*}{$\begin{array}{l}\text { Intención de adoptar } \\
\text { elearning }\end{array}$} & $\begin{array}{l}\text { Muy de } \\
\text { acuerdo }\end{array}$ & $\begin{array}{c}14 \\
82.4 \%\end{array}$ & $\begin{array}{c}29 \\
96.7 \%\end{array}$ & $\begin{array}{c}43 \\
91.5 \%\end{array}$ \\
\hline & & De acuerdo & $\begin{array}{c}3 \\
17.6 \%\end{array}$ & $\begin{array}{c}1 \\
3.3 \%\end{array}$ & $\begin{array}{c}4 \\
8.5 \%\end{array}$ \\
\hline & Total & & $\begin{array}{c}17 \\
100.0 \%\end{array}$ & $\begin{array}{c}30 \\
100.0 \%\end{array}$ & $\begin{array}{c}47 \\
100.0 \%\end{array}$ \\
\hline
\end{tabular}

De los estudiantes que tomaron entre uno y tres cursos en línea el $67 \%$ de los hombres estuvo muy de acuerdo y el $33.3 \%$ estuvo de acuerdo en aspectos como la motivación al estudiar en línea, el pensar positivamente acerca de la educación en línea; la intención de participar en cursos de educación en línea en el futuro, el disfrutar de aprender en esta modalidad y la posibilidad de continuar haciéndolo al mismo tiempo que recomendárselo a otros. Las mujeres en este grupo mostraron una actitud muy favorable con aproximadamente el $91 \%$ muy de acuerdo con los aspectos arriba mencionados. En el grupo de aquellos que tomaron más de 3 cursos en línea los resultados fueron aún más favorables con el 100\% de los hombres y el 93.8\% de las mujeres muy de acuerdo con la intención de utilizar la educación en línea y con los aspectos mencionados anteriormente.

En la tabla 3 se observa que el 100\% de la población de estudiantes, sin importar género o cantidad de cursos que han tomado en línea expresaron un total acuerdo con la facilidad de uso de los sistemas de educación en línea pues consideran que son fáciles de utilizar, que es fácil obtener recursos educativos de internet y también que cuando estudian en línea se hacen responsables de su propio aprendizaje. También mostraron a la una actitud positiva hacia la lectura en pantalla y la tecnología. 
Tabla 3

Distribución del nivel de acuerdo de los estudiantes hacia la facilidad de uso

\begin{tabular}{|c|c|c|c|c|c|}
\hline \multirow{2}{*}{ Género } & & & \multicolumn{2}{|c|}{$\begin{array}{c}\text { ¿Cuántos cursos ha tomado } \\
\text { en línea? }\end{array}$} & \multirow{2}{*}{ Total } \\
\hline & & & $\begin{array}{c}\text { De } 1 \text { a } 3 \\
\text { cursos }\end{array}$ & $\begin{array}{l}\text { Más de } 3 \\
\text { cursos }\end{array}$ & \\
\hline \multirow{4}{*}{ Masculino } & \multirow{2}{*}{ Facilidad de uso } & \multirow{4}{*}{ Muy de acuerdo } & 6 & 14 & 20 \\
\hline & & & $100.0 \%$ & $100.0 \%$ & $100.0 \%$ \\
\hline & \multirow{2}{*}{ Total } & & 6 & 14 & 20 \\
\hline & & & $100.0 \%$ & $100.0 \%$ & $100.0 \%$ \\
\hline \multirow{4}{*}{ Femenino } & \multirow{2}{*}{ Facilidad de uso } & \multirow{4}{*}{ Muy de acuerdo } & 11 & 16 & 27 \\
\hline & & & $100.0 \%$ & $100.0 \%$ & $100.0 \%$ \\
\hline & \multirow{2}{*}{ Total } & & 11 & 16 & 27 \\
\hline & & & $100.0 \%$ & $100.0 \%$ & $100.0 \%$ \\
\hline \multirow{3}{*}{ Total } & \multirow{2}{*}{ Facilidad de uso } & \multirow{2}{*}{ Muy de acuerdo } & 17 & 30 & 47 \\
\hline & & & $100.0 \%$ & $100.0 \%$ & $100.0 \%$ \\
\hline & Total & & $\begin{array}{c}17 \\
100.0 \%\end{array}$ & $\begin{array}{c}30 \\
100.0 \%\end{array}$ & $\begin{array}{c}47 \\
100.0 \%\end{array}$ \\
\hline
\end{tabular}

Tabla 4

Distribución del nivel de acuerdo de los estudiantes hacia el soporte pedagógico y técnico

\begin{tabular}{|c|c|c|c|c|c|}
\hline \multirow{2}{*}{ Género } & & & \multicolumn{2}{|c|}{$\begin{array}{c}\text { ¿Cuántos cursos ha tomado } \\
\text { en línea? }\end{array}$} & \multirow{2}{*}{ Total } \\
\hline & & & $\begin{array}{c}\text { De } 1 \text { a } 3 \\
\text { cursos }\end{array}$ & $\begin{array}{c}\text { Más de } 3 \\
\text { cursos }\end{array}$ & \\
\hline \multirow[t]{6}{*}{ Masculino } & Soporte pedagógico y & Muy de acuerdo & 5 & 14 & 19 \\
\hline & técnico & & $83.3 \%$ & $100.0 \%$ & $95.0 \%$ \\
\hline & & De acuerdo & 1 & 0 & 1 \\
\hline & & & $16.7 \%$ & $0.0 \%$ & $5.0 \%$ \\
\hline & Total & & 6 & 14 & 20 \\
\hline & & & $100.0 \%$ & $100.0 \%$ & $100.0 \%$ \\
\hline \multirow[t]{6}{*}{ Femenino } & Soporte pedagógico y & Muy de acuerdo & 11 & 15 & 26 \\
\hline & técnico & & $100.0 \%$ & $93.8 \%$ & $96.3 \%$ \\
\hline & & De acuerdo & 0 & 1 & 1 \\
\hline & & & $0.0 \%$ & $6.3 \%$ & $3.7 \%$ \\
\hline & Total & & 11 & 16 & 27 \\
\hline & & & $100.0 \%$ & $100.0 \%$ & $100.0 \%$ \\
\hline \multirow[t]{6}{*}{ Total } & Soporte pedagógico y & Muy de acuerdo & 16 & 29 & 45 \\
\hline & técnico & & $94.1 \%$ & $96.7 \%$ & $95.7 \%$ \\
\hline & & De acuerdo & 1 & 1 & 2 \\
\hline & & & $5.9 \%$ & $3.3 \%$ & $4.3 \%$ \\
\hline & Total & & 17 & 30 & 47 \\
\hline & & & $100.0 \%$ & $100.0 \%$ & $100.0 \%$ \\
\hline
\end{tabular}


En la tabla 4, en el caso de los hombres, el $83.3 \%$ de los que han tomado de uno a tres cursos en línea se mostraron muy de acuerdo con el soporte pedagógico y técnico recibido de parte de la institución proveedora de los cursos en línea y el restante 16.7\% mostraron una actitud de acuerdo. En el caso de los que tomaron más de tres cursos el 100\% de los hombres se mostró muy de acuerdo. Las mujeres que tomaron de uno a tres cursos en línea el 100\% se mostró muy de acuerdo y de las que tomaron más de tres cursos el $93.8 \%$ de las estudiantes se mostraron muy de acuerdo y el $6.8 \%$ de acuerdo. Es posible entonces observar que la mayoría de la población se encuentra muy de acuerdo en aspectos de apoyo tecnológico y técnico como; la universidad posee la tecnología necesaria para impartir cursos en línea, la interfaz de la institución es moderna y fácil de utilizar, la información se presenta en secuencia lógica y los tutores son entrenados y los motivan a utilizar los sistemas de educación en línea tanto para aprendizaje como para investigación.

Tabla 5

Distribución del nivel de acuerdo de los estudiantes hacia los estresores de la educación en línea

\begin{tabular}{|c|c|c|c|c|c|}
\hline \multirow{2}{*}{ Género } & & & \multicolumn{2}{|c|}{$\begin{array}{c}\text { ¿Cuántos cursos ha tomado en } \\
\text { línea? }\end{array}$} & \multirow{2}{*}{ Total } \\
\hline & & & $\begin{array}{c}\text { De } 1 \text { a } 3 \\
\text { cursos }\end{array}$ & $\begin{array}{l}\text { Más de } 3 \text { cur- } \\
\text { sos }\end{array}$ & \\
\hline \multirow[t]{2}{*}{ Masculino } & $\begin{array}{l}\text { Estresores de } \\
\text { elearning }\end{array}$ & Muy de acuerdo & $\begin{array}{c}6 \\
100.0 \%\end{array}$ & $\begin{array}{c}14 \\
100.0 \%\end{array}$ & $\begin{array}{c}20 \\
100.0 \%\end{array}$ \\
\hline & Total & & $\begin{array}{c}6 \\
100.0 \%\end{array}$ & $\begin{array}{c}14 \\
100.0 \%\end{array}$ & $\begin{array}{c}20 \\
100.0 \%\end{array}$ \\
\hline \multirow[t]{2}{*}{ Femenino } & $\begin{array}{l}\text { Estresores de } \\
\text { elearning }\end{array}$ & Muy de acuerdo & $\begin{array}{c}11 \\
100.0 \%\end{array}$ & $\begin{array}{c}16 \\
100.0 \%\end{array}$ & $\begin{array}{c}27 \\
100.0 \%\end{array}$ \\
\hline & Total & & $\begin{array}{c}11 \\
100.0 \%\end{array}$ & $\begin{array}{c}16 \\
100.0 \%\end{array}$ & $\begin{array}{c}27 \\
100.0 \%\end{array}$ \\
\hline \multirow[t]{2}{*}{ Total } & $\begin{array}{l}\text { Estresores de } \\
\text { elearning }\end{array}$ & Muy de acuerdo & $\begin{array}{c}17 \\
100.0 \%\end{array}$ & $\begin{array}{c}30 \\
100.0 \%\end{array}$ & $\begin{array}{c}47 \\
100.0 \%\end{array}$ \\
\hline & Total & & $\begin{array}{c}17 \\
100.0 \%\end{array}$ & $\begin{array}{c}30 \\
100.0 \%\end{array}$ & $\begin{array}{c}47 \\
100.0 \%\end{array}$ \\
\hline
\end{tabular}

En cuanto a aspectos que describen los estresores del aprendizaje en línea como ansiedad ante la propia habilidad de usar los sistemas virtuales, ansiedad ante las conexiones lentas a internet, la presión para utilizar la educación en línea y la posibilidad de que la educación en línea sea una opción para aquellos que trabajan, tienen familia o viven alejados geográficamente; el 100\% de la población de hombres y mujeres sin importar la cantidad de cursos que hubiesen recibido en línea indicaron una actitud positiva en estos aspectos mostrando una baja correlación entre el estrés y la educación en línea (ver tabla 5). 


\section{Discusión y conclusiones}

Los resultados encontrados indican que no existe una diferencia significativa entre la actitud hacia la educación en línea de alumnos que han tomado de uno a tres cursos en línea o más de tres, sin embargo, los que han tomado más de tres cursos tienen una actitud ligeramente más positiva que el otro grupo hacía continuar estudiando en modalidad virtual. Esto coincide con estudios anteriores que han encontrado una correlación positiva entre las habilidades técnicas y el uso de la tecnología, ya que a medida que el estudiante se familiarice con ellas el estrés disminuye y la motivación aumenta (Bertea, 2009). No se encontró una diferencia significativa entre las actitudes de hombres y mujeres.

Como se indicó anteriormente en estudios realizados por Bertea (2009), Pilli et al., (2014) y Saabah (2013) la experiencia previa con la tecnología, más no necesariamente con cursos virtuales, tiene una correlación positiva con la actitud de los estudiantes hacia la educación en línea. Esto concuerda con los resultados obtenidos en esta investigación en la cual la población tenía experiencia previa con cursos en línea y tecnología y mostraron una actitud positiva y motivación hacia esta modalidad de aprendizaje, que se manifestó en un acuerdo total hacia continuar estudiando en línea, una percepción positiva hacia la misma y la posibilidad de recomendarlo a otras personas. Los estudiantes mostraron también en su mayoría una actitud favorable hacia la percepción de que el aprendizaje en línea contribuye a mejorar la capacidad de razonamiento del estudiante lo que le permite ser más eficiente y productivo en los cursos, lo cual coincide con el estudio realizado por Mothibi (2015) quien encontró que el uso adecuado de los sistemas de TIC contribuye a mejorar el rendimiento académico del estudiante, si este tiene una percepción positiva de su uso. Los estudiantes se mostraron satisfechos con el soporte pedagógico y técnico de la institución y la facilidad de uso del sistema lo que se traduce en una mejor actitud hacia la intención de continuar utilizando los sistemas de aprendizaje en línea, esto coincide con lo encontrado por Pilli et al., (2014) quien refirió que si el estudiante está satisfecho con la calidad del sistema y su facilidad de uso tendrá una actitud positiva.

Se resalta que los estudiantes mostraron la actitud más favorable hacia la facilidad de uso de los sistemas de educación en línea, el soporte pedagógico y técnico de la institución proveedora de los cursos en línea, y hacia la posibilidad de que la educación en línea contribuya a reducir el estrés de aprender si se toman en cuenta factores como la accesibilidad y flexibilidad de los sistemas y la experiencia previa con la educación en línea y el uso de internet; vale la pena destacar que en cuanto a la percepción de utilidad de la educación en línea existe un área importante de oportunidad para las instituciones de educación superior, pues si bien es cierto que la mayoría de estudiantes mostraron una actitud favorable en este aspecto, una cantidad importante de estudiantes se encuentran aún indecisos acerca de si la educación en línea es más efectiva que otros sistemas de aprendizaje. Para los proveedores de cursos en línea esto es importante pues puede afectar la futura participación de algunos estudiantes indecisos. 
Se debe tomar en cuenta que los estudiantes que participaron en el estudio han tenido experiencia previa con el aprendizaje en línea, por lo que es de esperarse que su actitud sea positiva a la educación virtual. Waern y Ramberg (como se citó en Organista \& Backhoff, 2002), indican que el conocimiento previo y la experiencia computacional influyen en la percepción que el estudiante tenga acerca del aprendizaje que incorpora la computadora al proceso educativo.

Este estudio pone en evidencia que cuando una institución lleva a cabo iniciativas de aprendizaje en línea de calidad, accesible, flexible y fácil de utilizar, con un adecuado soporte de parte de la institución; hay como resultado una población de estudiantes con experiencia en computación motivados y dispuestos a aprovechar esta oferta de aprendizaje y beneficiarse de ella.

Fue posible determinar en el estudio que la mayoría de los estudiantes tenían de 46 años en adelante, lo cual podría indicar que esta oportunidad es aprovechada por estudiantes que tienen otras obligaciones familiares y laborales y han encontrado en la educación en línea la posibilidad de continuar sus estudios.

El fortalecimiento de la educación en línea es un reto importante para las instituciones actualmente. Las ventajas que ofrece para los estudiantes en cuanto a su flexibilidad, accesibilidad y facilidad de uso son importantes para aumentar el acceso a la educación y el desarrollo. Para que la educación en línea se convierta en un aliado importante de las instituciones educativas y del estudiante es importante considerar los factores actitudinales que pueden favorecer o interferir en la adopción de esta modalidad de enseñanza-aprendizaje. Este estudio se enfocó en la actitud hacia el aprendizaje en línea de estudiantes universitarios guatemaltecos con experiencia previa en esta modalidad. La investigación aporta un análisis e interpretación de las motivaciones y actitudes relacionadas con el aprendizaje en línea y se podrá utilizar para tomar decisiones en futuros programas virtuales. Será útil para tomar iniciativas que se adapten a las preferencias y conductas de los usuarios y mejores prácticas que provean a los estudiantes de un ambiente de aprendizaje en línea que aumente la motivación y satisfacción personal, lo que beneficiará directamente al estudiante en su desempeño académico y a la institución.

Este estudio es solamente un paso inicial, se realizó con estudiantes de una sola universidad, sin embargo, no existen indicadores de que los datos no puedan ser útiles a otras instituciones de educación superior que deseen tomar iniciativas de investigación similares.

Es necesario profundizar en el estudio de la actitud hacia el aprendizaje en línea en Guatemala para poder apoyar iniciativas educativas que contribuyan a que la educación llegue a todo aquel que la necesita traduciéndose en mayor desarrollo para el país. Se recomiendan estudios adicionales que tomen en cuenta la percepción del tutor hacia la educación en línea. También es recomendable llevar a cabo un estudio similar con una población que no ha tenido experiencia previa con la educación en línea ya que otros estudios indican que la actitud de los estudiantes hacia la educación en línea no es tan positiva cuando no están familiarizados con la tecnología y que su satisfacción aumenta con la experiencia, convirtiéndose para el estudiante en un recurso educativo útil (Tsenf, Chiang \& Hsu, como se citó en Alabdullaziz, et al., s.f.). 


\section{Referencias}

Ajzen, I. (1991). The theory of planned behavior. Organizational behavior and human decision processes, 50(2), 179-211. Recuperado de https://cas.hse.ru/data/816/479/1225/Oct\%20 19\%20Cited \%20\%231\%20Manage\%20THE\%20THEORY\%20OF\%20PLANNED\%20 BEHAVIOR.pdf

Alabdullaziz, F., Muhammad, M., Alyahya, S., \& Gall, J. (s.f.). Instructors' and learners' attitude toward e-learning within a college education. Department of Educational Technology. University of Northern Colorado. Recuperado de http:/files.eric. ed.gov/fulltext/ED528860.pd

Bertea, P. (2009). Measuring student's attitudes towards e-learning. A case study. Conferencie Proceedings of E-learning and Software in Education, (1), 417-424. Recuperado de https://www.ceeol.com/content-files/document-37597.pdf

Casal, J., \& Mateu, E. (2003). Tipos de muestreo. Rev. Epidem. Med. Prev, 1, 3-7. Recuperado de http://www.mat.uson.mx/ ftapia/Lecturas\%20Adicionales\%20 (Cómo\%20 diseñar\%20una\%20encuesta)/TiposMuestreo1.pdf

Centeno, G., \& Cubo, S. (2013). Evaluación de la competencia digital y las actitudes hacia las TIC del alumnado universitario. Revista de Investigación Educativa, 31(2), 517-536. http://dx.doi.org/10.6018/rie.31.2.169271

Elejabarrieta, F., \& Iñiguez, L. (2000). Construcción de escalas de actitud tipo Thurstone y Likert. Revista Electrónica La Sociología en sus Escenarios. Recuperado de http:// aprendeenlinea.udea.edu.co/revistas/index.php/ceo/article/view/6564

Hernández, R., Fernández, C., \& Baptista, M. (2014). Metodología de la Investigación. Recuperado de https://rabajosocialudocpno.files.wordpress.com/2017/07/metodolog c3a3c2ada_de_la_investigacic3a3c2b3n_-sampieri-_6ta_edicion1.pdf

Lee, L., \& Li, L. (2016). Computer literacy and online learning attitude toward GSOE students in distance education programs. Higher Education Studies, 6(3), 147-156. Recuperado de http://www.ccsenet.org/journal/index.php/hes/article/viewFile /61435/33419

Mehra, V., \& Omidian, F. (2012). Development an instrument to measure university students 'attitude towards e-learning. Turkish Online Journal of Distance Education. 13(1), 34-51. Recuperado de https://files.eric.ed.gov/fulltext/EJ976928.pdf

Morales, M. (2012). E-learning en Guatemala, perspectivas y oportunidades para instituciones educativas. El Observatorio de la Formación en Red. Boletín SCOPEO, (59). Recuperado de http://scopeo.usal.es/enfoque-bol-59-e-learning-en-guatemalaperspectivas-y-oportunidades-para-instituciones-educativas/

Mothibi, G. (2015). A meta- analysis of the relationship between e-learning and student's academic achievement in higher education. Journal of Education and Practice, 6(9), 6-9. Recuperado de http://files.eric.ed.gov/fulltext/EJ1082408.pdf

Orantes, S. (2011). Viabilidad del "Modelo de Aceptación de la Tecnología" en las empresas mexicanas. Una aproximación a las actitudes y percepciones de los usuarios de las tecnologías de la información. Revista.unam.mx, 12(1). Recuperado de http:// www.revista.unam.mx/vol.12/num1/art08/index.html

Organista, J., \& Backhoff, E. (2002). Opinión de estudiantes sobre el uso de apoyos didácticos en línea en un curso universitario. Revista Electrónica de Investigación Educativa, 4(1). Recuperado de https://redie.uabc.mx/redie/article/view/52/95 
Organización de las Naciones Unidas para la Educación, la Ciencia y la Cultura [UNESCO]. (2013). Enfoques estratégicos sobre las TICS en la educación en América Latina y el Caribe. Oficina Regional de educación para América Latina y el Caribe. Recuperado de http://www.unesco.org/new/fileadmin/MULTIMEDIA/FIELD/Santiago/images/ ticsesp.pdf

Oye, N., A.Iahad., N., Madar, M., \& Ab.Rahim, N., (2012). The Impact of e-learning on students' performance in tertiary institutions. International Journal of Computer Networks and Wireless Communications. 2(2), 121-130. Recuperado de http://www. ijcnwc.org/papers/vol2no22012/2vol2no2.pdf

Padilla, V., \& Rodríguez, M. (Noviembre, 2002). Conocimiento y actitudes de estudiantes universitarios hacia la educación virtual. Trabajo presentado en el Congreso Internacional de Educación para la Vida. UANL, Monterrey, N.L. México. Recuperado de http://www.academia.edu/2954645/Conocimiento_y_actitudes_de_estudiantes_universitarios_hacia_la_educaci\%C3\%B3n_virtual

Paris, P. (2004). E-Learning: A study on secondary students' attitudes towards online web Assisted Learning. International Education Journal, 5(1), 98-112. Recuperado de http://files.eric.ed.gov/fulltext/EJ903841.pdf

Pilli, O., Fanaeian, Y., \& Al-Momani, M. (2014). Investigating the students' attitude toward the use of e-learning in Girne American University. International Journal of Business and Social Science, 5(5), 169-175. Recuperado de http://ijbssnet.com /journals/ Vol_5_No_5_April_2014/19.pdf

Ruiz-Bolívar, C. (2002). Instrumentos de investigación educativa-procedimientos para su diseño y validación. Venezuela: CIDEG, C. A.

Saabah, N. (2013). Students' attitude and motivation towards e-learning. Presentado en el The First International Conference on Applied Sciences, Gaza-Palestine Recuperado de http://www.academia.edu/4765736/Students_Attitude_and_Motivation_Towards _E-learning

Yaghoubi, J., Mohammadi, I., Iravani, H., Attaran, M., \& Gheidi, A. (2008). Virtual student's perceptions of e-learning in Irán. The Turkish Online Journal of Education Technology, 7(3). Recuperado de http://files.eric.ed.gov/fulltext/ED502679.pdf

Fecha de recepción: 9 de diciembre de 2016

Fecha de revisión: 15 de diciembre de 2016

Fecha de aceptación: 7 de enero de 2018 